\title{
Heinz Type Inequalities for Poisson Integrals
}

\author{
Dariusz Partyka · Ken-ichi Sakan
}

Received: 27 September 2013 / Revised: 8 October 2013 / Accepted: 28 November 2013 /

Published online: 19 February 2014

(C) The Author(s) 2014. This article is published with open access at Springerlink.com

\begin{abstract}
In 1958, E. Heinz obtained a lower bound for $\left|\partial_{x} F\right|^{2}+\left|\partial_{y} F\right|^{2}$, where $F$ is a one-to-one harmonic mapping of the unit disk onto itself keeping the origin fixed. We show various variants of Heinz's inequality in the case where $F$ is the Poisson integral of a function of bounded variation in the unit circle. In particular, we obtain such inequalities for $F$ when it is a locally injective quasiregular mapping or an injective mapping of the unit disk onto a bounded convex domain in the complex plane.
\end{abstract}

Keywords Harmonic mappings · Poisson integral · Jacobian ·

Quasiconformal mappings · Quasiregular mappings

Mathematics Subject Classification (1991) Primary 30C55 · 30 C62

Communicated by Matti Vuorinen.

In memory of Professor Frederick William Gehring.

D. Partyka $(\bowtie)$

Institute of Mathematics and Computer Science, The John Paul II Catholic University of Lublin,

Al. Racławickie 14, P.O. Box 129, 20-950 Lublin, Poland

e-mail: partyka@kul.lublin.pl

D. Partyka

Institute of Mathematics and Information Technology, The State School of Higher Education in Chełm, Pocztowa 54, 22-100 Chełm, Poland

K. Sakan

Department of Mathematics, Graduate School of Science, Osaka City University, Sugimoto,

Sumiyoshi-ku, Osaka 558, Japan

e-mail:ksakan@sci.osaka-cu.ac.jp 


\section{Introduction}

Let $\mathbb{D}(a, r):=\{z \in \mathbb{C}:|z-a|<r\}, \overline{\mathbb{D}}(a, r):=\{z \in \mathbb{C}:|z-a| \leq r\}$ and $\mathbb{T}(a, r):=\{z \in \mathbb{C}:|z-a|=r\}$ for $a \in \mathbb{C}$ and $r>0$. In particular, $\mathbb{D}:=\mathbb{D}(0,1)$ and $\mathbb{T}:=\mathbb{T}(0,1)$ are the unit disk and unit circle, respectively. Assume that $F$ is a one-to-one harmonic mapping of $\mathbb{D}$ onto itself and normalised by $F(0)=0$. In 1958 , E. Heinz proved that the inequality

$$
\left|\partial_{x} F(z)\right|^{2}+\left|\partial_{y} F(z)\right|^{2} \geq \frac{2}{\pi^{2}}
$$

holds for every $z=x+\mathrm{i} y \in \mathbb{D}$; cf. [7]. Under certain additional assumptions on $F$ this inequality can be improved; cf. [11, Thm. 0.4, Thm. 0.6] and [12, Thm. 2.2, Cor. 2.4]. Given a function $f: \mathbb{T} \rightarrow \mathbb{C}$ and $z=\mathrm{e}^{\mathrm{i} \theta} \in \mathbb{T}$ we define

$$
\begin{aligned}
f^{\prime}(z) & :=\lim _{u \rightarrow z} \frac{f(u)-f(z)}{u-z}, \\
\dot{f}(z) & :=\lim _{t \rightarrow \theta} \frac{f\left(\mathrm{e}^{\mathrm{i} t}\right)-f\left(\mathrm{e}^{\mathrm{i} \theta}\right)}{t-\theta},
\end{aligned}
$$

provided the limits exist we also define $f^{\prime}(z):=0$ and $\dot{f}(z):=0$ otherwise. Obviously,

$$
\dot{f}(z)=\mathrm{i} z f^{\prime}(z) \text { and }|\dot{f}(z)|=\left|f^{\prime}(z)\right| \text {. }
$$

Recall that a function $f: \mathbb{T} \rightarrow \mathbb{C}$ is called Dini smooth if $f$ is differentiable on $\mathbb{T}$ and the derivative $\dot{f}$ is not vanishing, and Dini continuous on $\mathbb{T}$, i.e. its modulus of continuity

$$
\omega(\delta):=\sup \left\{\left|\dot{f}\left(\mathrm{e}^{\mathrm{i} t}\right)-\dot{f}\left(\mathrm{e}^{\mathrm{i} s}\right)\right|: t, s \in \mathbb{R},|t-s| \leq \delta\right\}, \quad \delta \in[0 ; 2 \pi],
$$

satisfies the following condition

$$
\int_{0}^{2 \pi} \frac{\omega(t)}{t} \mathrm{~d} t<+\infty
$$

We will use the standard notation $\partial:=\frac{1}{2}\left(\partial_{x}-\mathrm{i} \partial_{y}\right)$ and $\bar{\partial}:=\frac{1}{2}\left(\partial_{x}+\mathrm{i} \partial_{y}\right)$ for the so-called formal derivatives operators. The starting point of our work is the following two results obtained by the authors in 2009.

Theorem 1.1 ([13, Thm. 2.1]) Given an injective harmonic mapping $F$ of $\mathbb{D}$ onto a bounded convex domain $\Omega$ including 0 , assume that $F(0)=0,|\partial F(0)|-|\bar{\partial} F(0)|>0$ and that $F$ has a continuous extension to $\overline{\mathbb{D}}(0,1)$. If the boundary limiting valued function $f$ of $F$ is Dini smooth, then the following inequalities

$$
|\partial F(\zeta)| \geq \frac{R_{1}+R_{2}}{2 \pi} \tan \left(\frac{\pi}{2} \frac{R_{1}}{R_{1}+R_{2}}\right)+\frac{1}{2} \min _{z \in \mathbb{T}}\left|f^{\prime}(z)\right|
$$

as well as 


$$
\left|\partial_{x} F(\zeta)\right|^{2}+\left|\partial_{y} F(\zeta)\right|^{2} \geq 2\left(\frac{R_{1}+R_{2}}{2 \pi} \tan \left(\frac{\pi}{2} \frac{R_{1}}{R_{1}+R_{2}}\right)+\frac{1}{2} \min _{z \in \mathbb{T}}\left|f^{\prime}(z)\right|\right)^{2} .
$$

hold for every $\zeta \in \mathbb{D}$ and all $R_{1}, R_{2}>0$ satisfying $\mathbb{D}\left(0, R_{1}\right) \subset \Omega \subset \mathbb{D}\left(0, R_{2}\right)$.

Theorem 1.2 ([13, Thm. 2.2]) Given an injective harmonic mapping $F$ of $\mathbb{D}$ onto a bounded convex domain $\Omega$ including 0 , assume that $F(0)=0$ and $|\partial F(0)|-|\bar{\partial} F(0)|>$ 0 . Then for all $R_{1}, R_{2}>0$ satisfying $\mathbb{D}\left(0, R_{1}\right) \subset \Omega \subset \mathbb{D}\left(0, R_{2}\right)$ the following inequalities hold

$$
|\partial F(\zeta)| \geq \frac{R_{1}+R_{2}}{2 \pi} \tan \left(\frac{\pi}{2} \frac{R_{1}}{R_{1}+R_{2}}\right), \quad \zeta \in \mathbb{D}
$$

as well as

$$
\left|\partial_{x} F(\zeta)\right|^{2}+\left|\partial_{y} F(\zeta)\right|^{2} \geq 2\left(\frac{R_{1}+R_{2}}{2 \pi} \tan \left(\frac{\pi}{2} \frac{R_{1}}{R_{1}+R_{2}}\right)\right)^{2}, \quad \zeta \in \mathbb{D} .
$$

Note that if $\Omega=\mathbb{D}(0, R)$ for some $R>0$, then Theorem 1.2 implies the inequality

$$
\left|\partial_{x} F(\zeta)\right|^{2}+\left|\partial_{y} F(\zeta)\right|^{2} \geq \frac{2 R^{2}}{\pi^{2}}, \quad \zeta \in \mathbb{D},
$$

provided $F$ satisfies the assumptions of Theorem 1.2. In the case of the unit disk the inequality (1.9) coincides with the inequality (1.1), so the estimate (1.8) considerably extends the Heinz's classic one (1.1). The estimate (1.6) is even better provided $F$ is sufficiently regular at the boundary. On the other hand, Theorem 1.2 yields in the limiting case $R_{2} \rightarrow \infty$ Kalaj's inequality ([8, Thm. 2.5])

$$
\left|\partial_{x} F(\zeta)\right|^{2}+\left|\partial_{y} F(\zeta)\right|^{2} \geq \frac{1}{8} R_{1}^{2}, \quad \zeta \in \mathbb{D}
$$

as well as

$$
|\partial F(\zeta)| \geq \frac{R_{1}}{4}, \quad \zeta \in \mathbb{D},
$$

provided $\mathbb{D}\left(0, R_{1}\right) \subset F(\mathbb{D})$; cf. [13, Cor. 3.1].

If $f$ is an integrable function on $\mathbb{T}$, then we denote by $\mathrm{P}[f](\zeta)$ the Poisson integral of $f$ at $\zeta \in \mathbb{D}$, i.e.

$$
\mathrm{P}[f](\zeta):=\frac{1}{2 \pi} \int_{\mathbb{T}} f(u) \operatorname{Re} \frac{u+\zeta}{u-\zeta}|\mathrm{d} u|, \quad \zeta \in \mathbb{D}
$$

Here and in what follows integrable means integrable in the sense of Lebesgue. The Poisson integral $\mathrm{P}[f]$ is the unique solution to the Dirichlet problem for the unit disk $\mathbb{D}$ provided the boundary function $f$ is continuous; cf. e.g. [6, Thm. 2.11]. This means that $\mathrm{P}[f]$ is a harmonic mapping in $\mathbb{D}$ which has a continuous extension to the closed 
disk $\operatorname{cl}(\mathbb{D})$ and its boundary limiting valued function is identical with $f$. Here and later, $\operatorname{cl}(A)$ stands for the closure of a set $A \subset \mathbb{C}$ in the Euclidian topology. Therefore, we can rephrase Theorem 1.1 as follows.

Theorem 1.3 Given a Dini smooth function $f: \mathbb{T} \rightarrow \mathbb{C}$ assume that $F:=\mathrm{P}[f]$ is an injective mapping of $\mathbb{D}$ onto a convex domain $\Omega$ including $0, F(0)=0$ and $|\partial F(0)|-|\bar{\partial} F(0)|>0$. Then the inequalities (1.5) and (1.6) hold for every $\zeta \in \mathbb{D}$ and all $R_{1}, R_{2}>0$ satisfying $\mathbb{D}\left(0, R_{1}\right) \subset \Omega \subset \mathbb{D}\left(0, R_{2}\right)$.

Our main goal is to improve this theorem by dropping the assumption that $f$ is Dini smooth; cf. Theorem 4.4. We also present a number of related results dealing with estimates of Heinz type. In Sect. 2, we consider the general case of $f$ being of bounded variation; cf. Theorem 2.3 and Corollary 2.4. Section 3 deals with the case where $f$ is a regular mapping. Assuming that $\mathrm{P}[f](\mathbb{D})$ is a bounded convex domain we improve Theorems 1.3 and 1.1 by dropping the regularity of $f$. This is done in Sect. 4. The next section is devoted to study the subject under the assumption that $\mathrm{P}[f]$ is a locally injective quasiregular mapping, i.e. $\mathrm{P}[f]$ is locally injective and $K$-quasiregular for some $K \geq 1$, which means that

$$
|\bar{\partial} \mathrm{P}[f](\zeta)| \leq \frac{K-1}{K+1}|\partial \mathrm{P}[f](\zeta)|, \quad \zeta \in \mathbb{D} ;
$$

cf. e.g. [1, p. 25]. In the last section, we present a few applications of the earlier results.

\section{The General Case}

If $f$ is a function of bounded variation, then we write $\mathrm{P}[\mathrm{d} f](\zeta)$ for the Poisson-Stieltjes integral of $f$ at $\zeta \in \mathbb{D}$, i.e.

$$
\mathrm{P}[\mathrm{d} f](\zeta):=\frac{1}{2 \pi} \int_{\mathbb{T}} \operatorname{Re} \frac{u+\zeta}{u-\zeta} \mathrm{d} f(u), \quad \zeta \in \mathbb{D} .
$$

We recall that the harmonic conjugate operator $\mathrm{A}$ is defined for a function $f: \mathbb{T} \rightarrow \mathbb{C}$ integrable on $\mathbb{T}$ and $z \in \mathbb{T}$ as follows:

$$
\mathrm{A}[f](z):=\frac{1}{2 \pi} \lim _{r \rightarrow 1^{-}} \int_{0}^{2 \pi} f\left(\mathrm{e}^{\mathrm{i} t}\right) \operatorname{Im} \frac{\mathrm{e}^{\mathrm{i} t}+r z}{\mathrm{e}^{\mathrm{i} t}-r z} \mathrm{~d} t,
$$

whenever the limit exists and $\mathrm{A}[f](z):=0$ otherwise. It is known that for a.e. $z \in \mathbb{T}$ the limit exists; cf. [5, Ch. III, Lem. 1.1]. If $f$ is real valued, then the function

$$
\mathbb{D} \ni \zeta \mapsto \frac{1}{2 \pi} \int_{0}^{2 \pi} f\left(\mathrm{e}^{\mathrm{i} t}\right) \operatorname{Im} \frac{\mathrm{e}^{\mathrm{i} t}+\zeta}{\mathrm{e}^{\mathrm{i} t}-\zeta} \mathrm{d} t
$$


is the harmonic conjugate function of $\mathrm{P}[f]$, which justifies the name of the operator $\mathrm{A}$. Replacing $\mathrm{P}[f]$ by the Poisson-Stieltjes integral $\mathrm{P}[\mathrm{d} f]$ we may define the harmonic conjugate operator A for every function $f: \mathbb{T} \rightarrow \mathbb{C}$ of bounded variation and $z \in \mathbb{T}$ as follows:

$$
\mathrm{A}[\mathrm{d} f](z):=\frac{1}{2 \pi} \lim _{r \rightarrow 1^{-}} \int_{0}^{2 \pi} \operatorname{Im} \frac{\mathrm{e}^{\mathrm{i} t}+r z}{\mathrm{e}^{\mathrm{i} t}-r z} \mathrm{~d} f\left(\mathrm{e}^{\mathrm{i} t}\right),
$$

whenever the limit exists and $\mathrm{A}[\mathrm{d} f](z):=0$ otherwise. Note that

$$
\mathrm{A}[\mathrm{d} f](z)=\mathrm{A}[\dot{f}](z), \quad z \in \mathbb{T}
$$

provided $f$ is an absolutely continuous function. The following two lemmas are crucial for our considerations.

Lemma 2.1 Let $f: \mathbb{T} \rightarrow \mathbb{C}$ be a function of bounded variation and differentiable at a point $z \in \mathbb{T}$. If the limit $\lim _{r \rightarrow 1^{-}} \frac{\mathrm{d}}{\mathrm{d} r} \mathrm{P}[f](r z)$ exists, then the remaining limits in (2.5), (2.6) and (2.7) exist and

$$
\begin{aligned}
\mathrm{A}[\mathrm{d} f](z) & =\lim _{r \rightarrow 1^{-}} \frac{\mathrm{d}}{\mathrm{d} r} \mathrm{P}[f](r z) \\
& =\lim _{r \rightarrow 1^{-}} \frac{f(z)-\mathrm{P}[f](r z)}{1-r} \\
& =\lim _{r \rightarrow 1^{-}}(z \partial \mathrm{P}[f](r z)+\bar{z} \bar{\partial} \mathrm{P}[f](r z))
\end{aligned}
$$

as well as

$$
\begin{aligned}
\lim _{r \rightarrow 1^{-}} \partial \mathrm{P}[f](r z) & =\frac{\bar{z}}{2}\left(\mathrm{~A}[\mathrm{~d} f](z)+z f^{\prime}(z)\right), \\
\lim _{r \rightarrow 1^{-}} \bar{\partial} \mathrm{P}[f](r z) & =\frac{z}{2}\left(\mathrm{~A}[\mathrm{~d} f](z)-z f^{\prime}(z)\right) .
\end{aligned}
$$

Proof The lemma follows directly from [14, Lem. 1.1, Lem. 1.2].

For $p>0$, let $\mathrm{H}^{p}(\mathbb{D})$ stand for the Hardy space of holomorphic functions in the unit disk of the order p; cf. e.g. [3, Sec. 1.1].

Lemma 2.2 If $f: \mathbb{T} \rightarrow \mathbb{C}$ is a function of bounded variation, then $\partial \mathrm{P}[f], \overline{\bar{\partial} \mathrm{P}[f]} \in$ $\mathrm{H}^{p}(\mathbb{D})$ for every $p \in(0 ; 1)$ and the limit $\lim _{r \rightarrow 1^{-}} \frac{\mathrm{d}}{\mathrm{d} r} \mathrm{P}[f](r z)$ exists for a.e. $z \in \mathbb{T}$.

Proof The lemma follows directly from [14, Cor. 1.3].

Theorem 2.3 Given a function $f: \mathbb{T} \rightarrow \mathbb{C}$ of bounded variation assume that the mapping $F:=\mathrm{P}[f]$ is such that $\partial F(z) \neq 0$ for $z \in \mathbb{D}$ and

$$
\sup _{0<r<1} \int_{0}^{2 \pi}\left|\partial F\left(r \mathrm{e}^{\mathrm{i} \theta}\right)\right|^{-p} \mathrm{~d} \theta<+\infty
$$

for a certain $p>0$. Then for every $\zeta \in \mathbb{D}$, 


$$
|\partial F(\zeta)| \geq \frac{1}{2} \underset{z \in \mathbb{T}}{\operatorname{essinf}}\left(|\mathrm{A}[\mathrm{d} f](z)|^{2}+\left|f^{\prime}(z)\right|^{2}+2 \operatorname{Re}\left(\overline{z f^{\prime}(z)} \mathrm{A}[\mathrm{d} f](z)\right)\right)^{1 / 2}
$$

Proof Fix a function $f: \mathbb{T} \rightarrow \mathbb{C}$ satisfying the assumption. From Lemmas 2.1 and 2.2

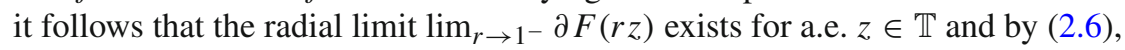

$$
\lim _{r \rightarrow 1^{-}}|\partial F(r z)| \geq \lambda \text { for a.e. } z \in \mathbb{T},
$$

where

$$
\lambda:=\frac{1}{2} \underset{z \in \mathbb{T}}{\operatorname{essinf}}\left(|\mathrm{A}[\mathrm{d} f](z)|^{2}+\left|f^{\prime}(z)\right|^{2}+2 \operatorname{Re}\left(\overline{z f^{\prime}(z)} \mathrm{A}[\mathrm{d} f](z)\right)\right)^{1 / 2} .
$$

If $\lambda=0$ then the inequality (2.9) is obvious. Thus we may assume that $\lambda>0$. By assumption, $\partial F$ is a holomorphic non-vanishing function in $\mathbb{D}$. Therefore, we may define the function $G:=(\partial F)^{-p}$. From (2.8) it follows that $G \in \mathrm{H}^{1}(\mathbb{D})$. Then there exists a Lebesgue integrable function $g: \mathbb{T} \rightarrow \mathbb{C}$ such that

$$
\lim _{r \rightarrow 1^{-}} G(r z)=g(z) \text { for a.e. } z \in \mathbb{T}
$$

and

$$
G(\zeta)=\mathrm{P}[g](\zeta), \quad \zeta \in \mathbb{D}
$$

cf. [3, Thm. 3.1]. Since $G^{-1 / p}=\partial F$ in $\mathbb{D}$, we deduce from (2.11) and (2.10) that

$$
|g(z)|^{-1 / p}=\lim _{r \rightarrow 1^{-}}|G(r z)|^{-1 / p}=\lim _{r \rightarrow 1^{-}}|\partial F(r z)| \geq \lambda \text { for a.e. } z \in \mathbb{T} .
$$

Hence and by (2.12),

$$
|\partial F(\zeta)|^{-p}=|G(\zeta)| \leq \mathrm{P}[|g|](\zeta) \leq \mathrm{P}\left[\lambda^{-p}\right](\zeta) \leq \lambda^{-p}, \quad \zeta \in \mathbb{D} .
$$

This implies the inequality (2.9), which completes the proof.

From Theorem 2.3, we can infer a number of lower estimates of $|\partial F|$ in $\mathbb{D}$. Let $\mathrm{J}[F]$ stand for the Jacobian of a differentiable mapping $F: \mathbb{D} \rightarrow \mathbb{C}$, i.e.

$$
\mathrm{J}[F](z):=|\partial F(z)|^{2}-|\bar{\partial} F(z)|^{2}, \quad z \in \mathbb{D} .
$$

Corollary 2.4 Given a function $f: \mathbb{T} \rightarrow \mathbb{C}$ of bounded variation assume that $F:=$ $\mathrm{P}[f]$ is a locally injective mapping in $\mathbb{D}, \mathrm{J}[F](0)>0$ and the condition (2.8) holds. Then for every $\zeta \in \mathbb{D}$, 


$$
\begin{aligned}
|\partial F(\zeta)| & \geq \frac{1}{2} \underset{z \in \mathbb{T}}{\operatorname{essinf}}\left(|\mathrm{A}[\mathrm{d} f](z)|^{2}+\left|f^{\prime}(z)\right|^{2}+2 \operatorname{Re}\left(\overline{z f^{\prime}(z)} \mathrm{A}[\mathrm{d} f](z)\right)\right)^{1 / 2} \\
& \geq \frac{1}{2} \underset{z \in \mathbb{T}}{\operatorname{essinf}}\left(|\mathrm{A}[\mathrm{d} f](z)|^{2}+\left|f^{\prime}(z)\right|^{2}\right)^{1 / 2} \\
& \geq \frac{1}{2} \underset{z \in \mathbb{T}}{\operatorname{essinf}}\left|f^{\prime}(z)\right|
\end{aligned}
$$

Proof Fix a function $f: \mathbb{T} \rightarrow \mathbb{C}$ satisfying the assumption. Since $F$ is a harmonic and locally injective mapping in $\mathbb{D}$, it follows from Lewy's theorem that the Jacobian $\mathrm{J}[F]$ does not vanish on $\mathbb{D}$; cf. [10]. Therefore,

$$
\mathrm{J}[F](z)=|\partial F(z)|^{2}-|\bar{\partial} F(z)|^{2}>0, \quad z \in \mathbb{D},
$$

because $\mathrm{J}[F](0)>0$. Hence,

$$
|\partial F(z)|>0, \quad z \in \mathbb{D} \text {. }
$$

Theorem 2.3 now yields the first inequality in (2.15). From Lemmas 2.1 and 2.2 it follows that the radial $\operatorname{limit}_{\lim } \rightarrow 1^{-} \partial F(r z)$ exists for a.e. $z \in \mathbb{T}$. Applying Lemma 2.1 we deduce from (2.6), (2.7) and (2.16) that for a.e. $z \in \mathbb{T}$,

$$
\operatorname{Re}\left(\overline{z f^{\prime}(z)} \mathrm{A}[\mathrm{d} f](z)\right)=\left|\lim _{r \rightarrow 1^{-}} \partial F(r z)\right|^{2}-\left|\lim _{r \rightarrow 1^{-}} \bar{\partial} F(r z)\right|^{2}=\lim _{r \rightarrow 1^{-}} \mathrm{J}[F](r z) \geq 0 .
$$

This shows the second inequality in (2.15). The last inequality in (2.15) is obvious, which proves the corollary.

\section{The Smooth Case}

In this section, we study the case where the function $f$ in Theorem 2.3 is fairly regular.

Theorem 3.1 Given a differentiable function $f: \mathbb{T} \rightarrow \mathbb{C}$ assume that $\dot{f}$ is Dini continuous, $F:=\mathrm{P}[f]$ is a locally injective mapping in $\mathbb{D}$ and $\mathrm{J}[F](0)>0$. Then the function $\mathrm{A}[\dot{f}]$ is continuous in $\mathbb{T}$ and for every $\zeta \in \mathbb{D}$,

$$
\begin{aligned}
|\partial F(\zeta)| & \geq \frac{1}{2} \min _{z \in \mathbb{T}}\left(|\mathrm{A}[\dot{f}](z)|^{2}+\left|f^{\prime}(z)\right|^{2}+2 \operatorname{Re}\left(\overline{z f^{\prime}(z)} \mathrm{A}[\dot{f}](z)\right)\right)^{1 / 2} \\
& \geq \frac{1}{2} \min _{z \in \mathbb{T}}\left(|\mathrm{A}[\dot{f}](z)|^{2}+\left|f^{\prime}(z)\right|^{2}\right)^{1 / 2} \\
& \geq \frac{1}{2} \min _{z \in \mathbb{T}}\left|f^{\prime}(z)\right| .
\end{aligned}
$$

Proof Fix a function $f: \mathbb{T} \rightarrow \mathbb{C}$ satisfying the assumption. Then $f$ is absolutely continuous and thus the equality (2.4) holds. From (1.12), it follows that for every $\zeta \in \mathbb{D} \backslash\{0\}$, 


$$
\begin{aligned}
\partial \mathrm{P}[f](\zeta) & =\frac{1}{2 \pi} \int_{\mathbb{T}} f(u) \frac{u}{(u-\zeta)^{2}}|\mathrm{~d} u| \\
& =-\frac{1}{2 \pi} \frac{1}{2 \mathrm{i} \zeta} \int_{0}^{2 \pi} f\left(\mathrm{e}^{\mathrm{i} t}\right) \frac{-2 \mathrm{i} \zeta \mathrm{e}^{\mathrm{i} t}}{\left(\mathrm{e}^{\mathrm{i} t}-\zeta\right)^{2}} \mathrm{~d} t \\
& =-\frac{1}{2 \pi} \frac{1}{2 \mathrm{i} \zeta} \int_{0}^{2 \pi} f\left(\mathrm{e}^{\mathrm{i} t}\right) \frac{\mathrm{d}}{\mathrm{d} t}\left(\frac{\mathrm{e}^{\mathrm{i} t}+\zeta}{\mathrm{e}^{\mathrm{i} t}-\zeta}\right) \mathrm{d} t,
\end{aligned}
$$

and consequently,

$$
\overline{\bar{\partial} \mathrm{P}[f](\zeta)}=\partial \overline{\mathrm{P}[f]}(\zeta)=\partial \mathrm{P}[\bar{f}](\zeta)=-\frac{1}{2 \pi} \frac{1}{2 \mathrm{i} \zeta} \int_{0}^{2 \pi} \overline{f\left(\mathrm{e}^{\mathrm{i} t}\right)} \frac{\mathrm{d}}{\mathrm{d} t}\left(\frac{\mathrm{e}^{\mathrm{i} t}+\zeta}{\mathrm{e}^{\mathrm{i} t}-\zeta}\right) \mathrm{d} t
$$

Integrating by parts we conclude from (3.2) and (3.3) that

$$
\begin{aligned}
& \zeta \partial F(\zeta)=\frac{1}{4 \pi \mathrm{i}} \int_{0}^{2 \pi} \dot{f}\left(\mathrm{e}^{\mathrm{i} t}\right) \frac{\mathrm{e}^{\mathrm{i} t}+\zeta}{\mathrm{e}^{\mathrm{i} t}-\zeta} \mathrm{d} t \\
& \zeta \overline{\bar{\partial} F(\zeta)}=\frac{1}{4 \pi \mathrm{i}} \int_{0}^{2 \pi} \overline{\dot{f}}\left(\mathrm{e}^{\mathrm{i} t}\right) \frac{\mathrm{e}^{\mathrm{i} t}+\zeta}{\mathrm{e}^{\mathrm{i} t}-\zeta} \mathrm{d} t .
\end{aligned}
$$

Note that the equalities (3.4) hold for $\zeta=0$, too. Since $\dot{f}$ is a Dini continuous function on $\mathbb{T}$, so is $\dot{\dot{f}}$. Applying now [15, Prop. 3.4] we conclude from the equalities (3.4) that both the functions $\partial F$ and $\bar{\partial} F$ have continuous extensions $F_{1}$ and $F_{2}$ to the closure $\operatorname{cl}(\mathbb{D})$, respectively. Since

$$
\frac{\mathrm{d}}{\mathrm{d} r} \mathrm{P}[f](r z)=z \partial F(r z)+\bar{z} \bar{\partial} F(r z)=z F_{1}(r z)+\bar{z} F_{2}(r z), \quad z \in \mathbb{T},
$$

the $\operatorname{limit}_{\lim _{r \rightarrow 1^{-}}} \frac{\mathrm{d}}{\mathrm{d} r} \mathrm{P}[f](r z)$ exists for every $z \in \mathbb{T}$, and by Lemma 2.1,

$$
\mathrm{A}[\dot{f}](z)=\mathrm{A}[\mathrm{d} f](z)=\lim _{r \rightarrow 1^{-}} \frac{\mathrm{d}}{\mathrm{d} r} \mathrm{P}[f](r z)=z F_{1}(z)+\bar{z} F_{2}(z), \quad z \in \mathbb{T} .
$$

Hence, $\mathrm{A}[\dot{f}]$ is a continuous function in $\mathbb{T}$.

Suppose that $F_{1}(z)=0$ for a certain $z \in \mathbb{T}$. By (2.16), $|\bar{\partial} F(r z)|^{2}<|\partial F(r z)|^{2}$ for $r \in[0 ; 1)$, and so

$$
\left|F_{2}(z)\right|^{2}=\lim _{r \rightarrow 1^{-}}\left|F_{2}(r z)\right|^{2} \leq \lim _{r \rightarrow 1^{-}}\left|F_{1}(r z)\right|^{2}=\left|F_{1}(z)\right|^{2}=0 .
$$


Thus $F_{2}(z)=0$, and by (2.6) and (2.7) we obtain $f^{\prime}(z)=0$ and $\mathrm{A}[\dot{f}](z)=0$. This clearly forces the inequalities (3.1). Therefore, we may assume that $\left|F_{1}(z)\right|>0$ for every $z \in \mathbb{T}$. From this and (2.17) we see that

$$
\left|F_{1}(\zeta)\right|>0, \quad \zeta \in \operatorname{cl}(\mathbb{D})
$$

Since the function $F_{1}$ is continuous in the compact set $\operatorname{cl}(\mathbb{D})$, we conclude that $1 / \partial F$ is a bounded function in $\mathbb{D}$. Therefore, the condition $(2.8)$ holds for $p:=1$. Since both the functions $f^{\prime}$ and $\mathrm{A}[\dot{f}]$ are continuous in $\mathbb{T}$, we infer from Corollary 2.4 the inequalities (3.1), which completes the proof.

Corollary 3.2 Let $F: \mathbb{D} \rightarrow \mathbb{C}$ be a locally injective harmonic mapping such that $\mathrm{J}[F](0)>0$. Then for every $R \in(0 ; 1)$,

$$
\begin{aligned}
|\partial F(\zeta)| & \geq \frac{1}{2} \min _{z \in \mathbb{T}(0, R)}\left(\left|\frac{\partial F(z)}{\partial r}\right|^{2}+\frac{1}{R^{2}}\left|\frac{\partial F(z)}{\partial \theta}\right|^{2}-\frac{2}{R} \operatorname{Im}\left(\frac{\partial F(z)}{\partial \theta} \frac{\partial F(z)}{\partial r}\right)\right)^{1 / 2} \\
& \geq \frac{1}{2} \min _{z \in \mathbb{T}(0, R)}\left(\left|\frac{\partial F(z)}{\partial r}\right|^{2}+\frac{1}{R^{2}}\left|\frac{\partial F(z)}{\partial \theta}\right|^{2}\right)^{1 / 2} \\
& \geq \frac{1}{2 R} \min _{z \in \mathbb{T}(0, R)}\left|\frac{\partial F(z)}{\partial \theta}\right|, \quad \zeta \in \mathbb{D}(0, R)
\end{aligned}
$$

where $\partial F / \partial r$ and $\partial F / \partial \theta$ denote the partial derivatives with respect to polar coordinates $(0 ;+\infty) \times \mathbb{R} \ni(r, \theta) \mapsto r \mathrm{e}^{\mathrm{i} \theta}$.

Proof Fix a function $F: \mathbb{D} \rightarrow \mathbb{C}$ satisfying the assumption. Given $R \in(0 ; 1)$ we define the function $\mathbb{T} \ni z \mapsto f(z):=F(R z)$. Since the function $\mathbb{D} \ni \zeta \mapsto F(R \zeta)$ is harmonic in $\mathbb{D}$ and the function $f$ is continuous in $\mathbb{T}$, we see that

$$
F(R \zeta)=\mathrm{P}[f](\zeta), \quad \zeta \in \mathbb{D}
$$

Hence,

$$
\partial F(R \zeta)=\frac{1}{R} \partial \mathrm{P}[f](\zeta), \quad \zeta \in \mathbb{D}
$$

From (1.3) it follows that for every $z=\mathrm{e}^{\mathrm{i} \theta} \in \mathbb{T}$,

$$
\dot{f}(z)=\lim _{t \rightarrow \theta} \frac{f\left(\mathrm{e}^{\mathrm{i} t}\right)-f\left(\mathrm{e}^{\mathrm{i} \theta}\right)}{t-\theta}=\lim _{t \rightarrow \theta} \frac{F\left(R \mathrm{e}^{\mathrm{i} t}\right)-F\left(R \mathrm{e}^{\mathrm{i} \theta}\right)}{t-\theta}=\frac{\partial F(R z)}{\partial \theta} .
$$

By (3.7) and (2.5) we have

$$
\begin{aligned}
\mathrm{A}[\dot{f}](z) & =\mathrm{A}[\mathrm{d} f](z)=\lim _{r \rightarrow 1^{-}} \frac{f(z)-\mathrm{P}[f](r z)}{1-r} \\
& =R \lim _{r \rightarrow 1^{-}} \frac{F(R z)-F(R r z)}{R-R r}=R \frac{\partial F(R z)}{\partial r} .
\end{aligned}
$$


By the regularity of $F$ we see that $\dot{f}$ is a Dini continuous function in $\mathbb{T}$. By the assumption and (3.7) it follows that $\mathrm{P}[f]$ is a locally injective mapping. Applying now Theorem 3.1 we infer from (3.8), (3.9) and (3.10) the inequalities in (3.6) for any $\zeta \in \mathbb{D}(0, R)$, which proves the corollary.

\section{The Case of a Mapping of Bounded Convex Image}

We now focus our attention to Poisson integrals mapping the unit disk onto bounded convex domains. We will enhance Theorem 1.3.

Lemma 4.1 Let $f: \mathbb{T} \rightarrow \mathbb{C}$ be a function of bounded variation and differentiable at a point $z \in \mathbb{T}$ such that $\mathrm{P}[f](0)=0 \neq \mathrm{J}[\mathrm{P}[f]](0)$, the limit $\lim _{r \rightarrow 1^{-}} \frac{\mathrm{d}}{\mathrm{d} r} \mathrm{P}[f](r z)$ exists and

$$
\liminf _{r \rightarrow 1^{-}} \mathrm{J}[\mathrm{P}[f]](r z) \geq 0 .
$$

If $\xi \in \mathbb{T}$ satisfies the condition

$$
\operatorname{Re}(\xi \mathrm{P}[f](u)) \leq \operatorname{Re}(\xi f(z)), \quad u \in \mathbb{D},
$$

then the following limits exist and

$$
\lim _{r \rightarrow 1^{-}} \operatorname{Re} \frac{\xi f(z)-\xi \mathrm{P}[f](r z)}{1-r} \geq \frac{a+b}{\pi} \tan \left(\frac{\pi}{2} \frac{\min (a, b)}{a+b}\right) \geq \frac{\min (a, b)}{2}
$$

as well as

$$
\begin{aligned}
\lim _{r \rightarrow 1^{-}} \mathrm{J}[\mathrm{P}[f]](r z) & =\left|f^{\prime}(z)\right| \lim _{r \rightarrow 1^{-}} \operatorname{Re} \frac{\xi f(z)-\xi \mathrm{P}[f](r z)}{1-r} \\
& \geq\left|f^{\prime}(z)\right| \frac{a+b}{\pi} \tan \left(\frac{\pi}{2} \frac{\min (a, b)}{a+b}\right) \geq\left|f^{\prime}(z)\right| \frac{\min (a, b)}{2},
\end{aligned}
$$

where

$$
a:=-\inf _{u \in \mathbb{D}} \operatorname{Re}(\xi \mathrm{P}[f](u)) \text { and } b:=\sup _{u \in \mathbb{D}} \operatorname{Re}(\xi \mathrm{P}[f](u)) .
$$

Proof The lemma follows directly from [14, Lem. 2.3, Lem. 2.4].

Remark 4.2 The condition (4.2) means geometrically that there exists a closed half plane $H$ such that its boundary line passes through the point $f(z)$ and $\Omega:=\mathrm{P}[f](\mathbb{D}) \subset$ $H$. Therefore, the point $f(z)$ is said to be linearly accessible from outside of $\Omega$. If $\Omega$ is a convex domain in $\mathbb{C}$, then each point $v \in \mathbb{C} \backslash \Omega$ is linearly accessible from outside of $\Omega$, i.e. there exists $\xi \in \mathbb{T}$ such that

$$
\operatorname{Re}(\xi w) \leq \operatorname{Re}(\xi v), \quad w \in \Omega
$$

cf. [14, Def. 2.1, Rem. 2.2]. In particular, if $\Omega$ is a convex domain in $\mathbb{C}$, then each point $z \in \mathbb{T}$ such that $f(z) \notin \Omega$ satisfies the condition (4.2) for a certain $\xi \in \mathbb{T}$. 
Lemma 4.3 Let $f: \mathbb{T} \rightarrow \mathbb{C}$ be a function of bounded variation and differentiable at a point $z \in \mathbb{T}$ such that $\mathrm{P}[f](0)=0 \neq \mathrm{J}[\mathrm{P}[f]](0)$, the limit $\lim _{r \rightarrow 1^{-}} \frac{\mathrm{d}}{\mathrm{d} r} \mathrm{P}[f](r z)$ exists and the inequality (4.1) holds. If there exists $\xi \in \mathbb{T}$ satisfying the condition (4.2), then

$$
|\mathrm{A}[\mathrm{d} f](z)| \geq \frac{a+b}{\pi} \tan \left(\frac{\pi}{2} \frac{\min (a, b)}{a+b}\right) \geq \frac{\min (a, b)}{2}
$$

and

$$
\operatorname{Re}\left(\overline{z f^{\prime}(z)} \mathrm{A}[\mathrm{d} f](z)\right) \geq\left|f^{\prime}(z)\right| \frac{a+b}{\pi} \tan \left(\frac{\pi}{2} \frac{\min (a, b)}{a+b}\right) \geq\left|f^{\prime}(z)\right| \frac{\min (a, b)}{2},
$$

where the constants $a$ and $b$ are given by (4.5). In particular,

$$
|\mathrm{A}[\mathrm{d} f](z)| \geq \frac{R_{1}+R_{2}}{\pi} \tan \left(\frac{\pi}{2} \frac{R_{1}}{R_{1}+R_{2}}\right) \geq \frac{R_{1}}{2}
$$

and

$$
\operatorname{Re}\left(\overline{z f^{\prime}(z)} \mathrm{A}[\mathrm{d} f](z)\right) \geq\left|f^{\prime}(z)\right| \frac{R_{1}+R_{2}}{\pi} \tan \left(\frac{\pi}{2} \frac{R_{1}}{R_{1}+R_{2}}\right) \geq\left|f^{\prime}(z)\right| \frac{R_{1}}{2}
$$

for all $R_{1}, R_{2}>0$ satisfying

$$
\mathbb{D}\left(0, R_{1}\right) \subset \mathrm{P}[f](\mathbb{D}) \subset \mathbb{D}\left(0, R_{2}\right) .
$$

Proof Fix $f$ and $z$ satisfying the assumption and assume that there exists $\xi \in \mathbb{T}$ satisfying the condition (4.2). From (2.5) and (4.3) it follows that

$$
\begin{aligned}
|\mathrm{A}[\mathrm{d} f](z)| & =\lim _{r \rightarrow 1^{-}}\left|\frac{f(z)-\mathrm{P}[f](r z)}{1-r}\right| \geq \lim _{r \rightarrow 1^{-}}\left|\operatorname{Re} \frac{\xi f(z)-\xi \mathrm{P}[f](r z)}{1-r}\right| \\
& \geq \frac{a+b}{\pi} \tan \left(\frac{\pi}{2} \frac{\min (a, b)}{a+b}\right) \geq \frac{\min (a, b)}{2},
\end{aligned}
$$

which gives (4.7). From (2.6), (2.7) and (4.4) it follows that

$$
\begin{aligned}
\operatorname{Re}\left(\overline{z f^{\prime}(z)} \mathrm{A}[\mathrm{d} f](z)\right) & =\left|\frac{\bar{z}}{2}\left(\mathrm{~A}[\mathrm{~d} f](z)+z f^{\prime}(z)\right)\right|^{2}-\left|\frac{\bar{z}}{2}\left(\mathrm{~A}[\mathrm{~d} f](z)-z f^{\prime}(z)\right)\right|^{2} \\
& =\lim _{r \rightarrow 1^{-}}|\partial \mathrm{P}[f](r z)|^{2}-\lim _{r \rightarrow 1^{-}}|\bar{\partial} \mathrm{P}[f](r z)|^{2}=\lim _{r \rightarrow 1^{-}} \mathrm{J}[\mathrm{P}[f]](r z) \\
& \geq\left|f^{\prime}(z)\right| \frac{a+b}{\pi} \tan \left(\frac{\pi}{2} \frac{\min (a, b)}{a+b}\right) \geq\left|f^{\prime}(z)\right| \frac{\min (a, b)}{2},
\end{aligned}
$$

which leads to (4.8).

Fix $R_{1}, R_{2}>0$ satisfying the condition (4.11). From (4.2), (4.5) and (4.11) we conclude that $R_{1} \leq a \leq R_{2}$ and $R_{1} \leq b \leq R_{2}$. Hence, $\max (a / b, b / a) \leq R_{2} / R_{1}$. Since $(0 ; \pi / 2) \ni t \mapsto t^{-1} \tan t$ is an increasing function, we derive from (4.7) and (4.8) the inequalities (4.9) and (4.10), respectively, which completes the proof. 
For a function $f: \mathbb{T} \rightarrow \mathbb{C}$ of bounded variation we define

$$
d_{f}:=\underset{z \in \mathbb{T}}{\operatorname{essinf}}\left|f^{\prime}(z)\right|
$$

Theorem 4.4 Given a function $f: \mathbb{T} \rightarrow \mathbb{C}$ of bounded variation assume that $F:=$ $\mathrm{P}[f]$ is an injective mapping in $\mathbb{D}$ and $\mathrm{J}[F](0)>0$. If $\Omega:=F(\mathbb{D})$ is a bounded convex domain, then

$$
|\partial F(\zeta)| \geq \frac{R_{1}+R_{2}}{2 \pi} \tan \left(\frac{\pi}{2} \frac{R_{1}}{R_{1}+R_{2}}\right)+\frac{1}{2} d_{f} \geq \frac{R_{1}}{4}+\frac{1}{2} d_{f}, \quad \zeta \in \mathbb{D}
$$

for all $R_{1}, R_{2}>0$ satisfying

$$
\mathbb{D}\left(F(0), R_{1}\right) \subset F(\mathbb{D}) \subset \mathbb{D}\left(F(0), R_{2}\right) .
$$

Proof Given $f: \mathbb{T} \rightarrow \mathbb{C}$ satisfying the assumptions fix $R_{1}, R_{2}>0$ such that the condition (4.14) holds. Suppose that $F(0)=0$. Then the condition (4.14) coincides with (4.11). Since $\mathrm{J}[F](0)>0$, we deduce from Lewy's theorem $([10])$ that $\mathrm{J}[F](\zeta)>$ 0 for $\zeta \in \mathbb{D}$, and so the condition (4.1) holds for every $z \in \mathbb{T}$. Since the function $f$ is of bounded variation, there exists the derivative $f^{\prime}(z)$ for a.e. $z \in \mathbb{T}$. From Lemma 2.2

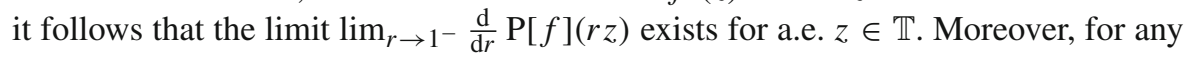
point $z \in \mathbb{T}$ where $f$ is differentiable, $f$ is continuous at $z$, and so $F(\zeta) \rightarrow f(z)$ as $\mathbb{D} \ni \zeta \rightarrow z$. From this and the injectivity of $F$ it follows that $f(z) \notin \Omega$. Since $\Omega$ is a bounded convex domain, we see by Remark 4.2 that the point $z$ satisfies the condition (4.2) for a certain $\zeta \in \mathbb{T}$. Therefore, the assumptions of Lemma 4.3 hold for a.e. $z \in \mathbb{T}$. By Theorem 1.2, the inequality (1.7) holds for every $\zeta \in \mathbb{D}$. Thus $1 / \partial F$ is a bounded function, and consequently the condition (2.8) is satisfied for any $p>0$. Applying now Corollary 2.4 we deduce from (4.9) and (4.10) the first inequality in (4.13) for every $\zeta \in \mathbb{D}$. The second inequality in (4.13) follows directly from the inequality $\tan x \geq x$ for $x \in[0 ; \pi / 2)$.

If $a:=F(0) \neq 0$ we can replace $f$ by $f_{a}:=f-a$. Then the function $f_{a}$ is of bounded variation. Moreover, $F_{a}:=\mathrm{P}\left[f_{a}\right]=\mathrm{P}[f]-\mathrm{P}[a]=F-a$ in $\mathbb{D}$, and so $F_{a}(0)=0, F_{a}$ is an injective mapping in $\mathbb{D}, \mathrm{J}\left[F_{a}\right](0)=\mathrm{J}[F](0)>0$ and $F_{a}(\mathbb{D})$ is a bounded convex domain. Therefore, the mapping $F_{a}$ satisfies the inequality in (4.13) for every $\zeta \in \mathbb{D}$ with $F$ and $f$ replaced by $F_{a}$ and $f_{a}$, respectively. Since $\partial F_{a}=\partial F$ in $\mathbb{D}$ and $f^{\prime}=f_{a}^{\prime}$ in $\mathbb{T}$ we obtain the estimate (4.13) without the assumption $F(0)=0$, which completes the proof.

\section{The Case of Quasiregularity}

We are able to improve estimates which are obtained so far, provided the Poisson integral $\mathrm{P}[f]$ is a quasiregular mapping. It will be done by employing the following lemma. 
Lemma 5.1 Given $K \geq 1$ and a function $f: \mathbb{T} \rightarrow \mathbb{C}$ of bounded variation assume that $F:=\mathrm{P}[f]$ is a $K$-quasiregular mapping. Then

$$
\frac{1}{K}\left|f^{\prime}(z)\right| \leq|\mathrm{A}[\mathrm{d} f](z)| \leq K\left|f^{\prime}(z)\right|
$$

for a.e. $z \in \mathbb{T}$, to be specific, for $z \in \mathbb{T}$ where $f$ is differentiable and the limit $\lim _{r \rightarrow 1^{-}} \frac{\mathrm{d}}{\mathrm{d} r} \mathrm{P}[f](r z)$ exists.

Proof Fix $K \geq 1$ and a function $f: \mathbb{T} \rightarrow \mathbb{C}$ satisfying the assumptions. Let $z \in \mathbb{T}$ be a point where $f$ is differentiable and the limit $\lim _{r \rightarrow 1^{-}} \frac{\mathrm{d}}{\mathrm{d} r} \mathrm{P}[f](r z)$ exists. Since $F$ is a $K$-quasiregular mapping, we conclude from Lemma 2.1 that

$$
\begin{aligned}
\left|f^{\prime}(z)\right| & =\lim _{r \rightarrow 1^{-}}|z \partial F(r z)-\bar{z} \bar{\partial} F(r z)| \geq \lim _{r \rightarrow 1^{-}}(|\partial F(r z)|-|\bar{\partial} F(r z)|) \\
& \geq \frac{1}{K} \lim _{r \rightarrow 1^{-}}(|\partial F(r z)|+|\bar{\partial} F(r z)|) \geq \frac{1}{K} \lim _{r \rightarrow 1^{-}}(|z \partial F(r z)+\bar{z} \bar{\partial} F(r z)|) \\
& =\frac{1}{K}|\mathrm{~A}[\mathrm{~d} f](z)|,
\end{aligned}
$$

which implies the second inequality in (5.1). Likewise,

$$
\begin{aligned}
\left|f^{\prime}(z)\right| & =\lim _{r \rightarrow 1^{-}}|z \partial F(r z)-\bar{z} \bar{\partial} F(r z)| \leq \lim _{r \rightarrow 1^{-}}(|\partial F(r z)|+|\bar{\partial} F(r z)|) \\
& \leq K \lim _{r \rightarrow 1^{-}}(|\partial F(r z)|-|\bar{\partial} F(r z)|) \leq K \lim _{r \rightarrow 1^{-}}(|z \partial F(r z)+\bar{z} \bar{\partial} F(r z)|) \\
& =K|\mathrm{~A}[\mathrm{~d} f](z)|,
\end{aligned}
$$

which implies the first inequality in (5.1).

The following theorem corresponds to Corollary 2.4 .

Theorem 5.2 Given $K \geq 1$ and a function $f: \mathbb{T} \rightarrow \mathbb{C}$ of bounded variation assume that $F:=\mathrm{P}[f]$ is a locally injective $K$-quasiregular mapping and the condition (2.8) holds. Then for every $\zeta \in \mathbb{D}$,

$$
\begin{aligned}
|\partial F(\zeta)| & \geq \frac{\sqrt{K^{2}+4 K+1}}{2(K+1)} \underset{z \in \mathbb{T}}{\operatorname{essinf}}\left(|\mathrm{A}[\mathrm{d} f](z)|^{2}+\left|f^{\prime}(z)\right|^{2}\right)^{1 / 2} \\
& \geq \frac{\sqrt{\left(K^{2}+4 K+1\right)\left(K^{2}+1\right)}}{2(K+1) K} d_{f} .
\end{aligned}
$$

Proof Fix $K \geq 1$ and a function $f: \mathbb{T} \rightarrow \mathbb{C}$ satisfying the assumptions. Since the mapping $F$ is $K$-quasiregular, we see by (1.13) that

$$
|\bar{\partial} F(\zeta)| \leq \frac{K-1}{K+1}|\partial F(\zeta)|, \quad \zeta \in \mathbb{D} .
$$


Hence for every $\zeta \in \mathbb{D}$,

$$
|\mathrm{J}[F](\zeta)|=|\partial F(\zeta)|^{2}-|\bar{\partial} F(\zeta)|^{2} \geq|\partial F(\zeta)|^{2}\left(1-\frac{(K-1)^{2}}{(K+1)^{2}}\right)=\frac{4 K|\partial F(\zeta)|^{2}}{(K+1)^{2}}
$$

Applying now Lemmas 2.1 and 2.2 we deduce from (2.6) and (2.7) that for a.e. $z \in \mathbb{T}$,

$$
\begin{aligned}
\operatorname{Re}\left(\overline{z f^{\prime}(z)} \mathrm{A}[\mathrm{d} f](z)\right) & =\left|\lim _{r \rightarrow 1^{-}} \partial F(r z)\right|^{2}-\left|\lim _{r \rightarrow 1^{-}} \bar{\partial} F(r z)\right|^{2} \\
& =\lim _{r \rightarrow 1^{-}} \mathrm{J}[F](r z) \geq \lim _{r \rightarrow 1^{-}} \frac{4 K|\partial F(r z)|^{2}}{(K+1)^{2}}
\end{aligned}
$$

Setting

$$
\lambda:=\underset{z \in \mathbb{T}}{\operatorname{essinf}}\left(|\mathrm{A}[\mathrm{d} f](z)|^{2}+\left|f^{\prime}(z)\right|^{2}\right)^{1 / 2}
$$

we conclude from Corollary 2.4 and (5.4) that for every $\zeta \in \mathbb{D}$,

$$
\begin{aligned}
|\partial F(\zeta)|^{2} & \geq \frac{1}{4} \underset{z \in \mathbb{T}}{\operatorname{essinf}}\left(|\mathrm{A}[\mathrm{d} f](z)|^{2}+\left|f^{\prime}(z)\right|^{2}+2 \operatorname{Re}\left(\overline{z f^{\prime}(z)} \mathrm{A}[\mathrm{d} f](z)\right)\right) \\
& \geq \frac{\lambda^{2}}{4}+\frac{1}{2} \underset{z \in \mathbb{T}}{\operatorname{essinf}} \operatorname{Re}\left(\overline{z f^{\prime}(z)} \mathrm{A}[\mathrm{d} f](z)\right) \\
& \geq \frac{\lambda^{2}}{4}+\frac{1}{2} \underset{z \in \mathbb{T}}{\operatorname{essinf}} \liminf _{r \rightarrow 1^{-}} \frac{4 K|\partial F(r z)|^{2}}{(K+1)^{2}} \\
& \geq \frac{\lambda^{2}}{4}+\frac{1}{2} \frac{\lambda^{2} K}{(K+1)^{2}}=\lambda^{2} \frac{(K+1)^{2}+2 K}{4(K+1)^{2}}
\end{aligned}
$$

This yields the first inequality in (5.2). The second inequality in (5.2) follows from Lemma 5.1.

The next theorem corresponds to Theorem 3.1.

Theorem 5.3 Given $K \geq 1$ and a differentiable function $f: \mathbb{T} \rightarrow \mathbb{C}$ assume that $\dot{f}$ is Dini continuous and $F:=\mathrm{P}[f]$ is a locally injective $K$-quasiregular mapping. Then the function $\mathrm{A}(\dot{f})$ is continuous in $\mathbb{T}$ and for every $\zeta \in \mathbb{D}$,

$$
\begin{aligned}
|\partial F(\zeta)| & \geq \frac{\sqrt{K^{2}+4 K+1}}{2(K+1)} \min _{z \in \mathbb{T}}\left(|\mathrm{A}[\dot{f}](z)|^{2}+\left|f^{\prime}(z)\right|^{2}\right)^{1 / 2} \\
& \geq \frac{\sqrt{\left(K^{2}+4 K+1\right)\left(K^{2}+1\right)}}{2(K+1) K} \min _{z \in \mathbb{T}}\left|f^{\prime}(z)\right| .
\end{aligned}
$$

Proof The proof runs in much the same way as the proof of Theorem 5.2. The only difference is that we use Theorem 3.1 instead of Corollary 2.4. 


\section{Applications}

In this section, we provide a few applications of the results obtained in the previous sections.

Remark 6.1 All the estimates (2.9), (2.15), (3.1), (3.6), (4.13) and (5.2) are applicable under the assumption that $F$ is a harmonic mapping of $\mathbb{D}$ onto a Jordan domain $\Omega$ bounded by a rectifiable Jordan curve $\Gamma$, which has a continuous and injective extension $F^{*}$ to the closure $\operatorname{cl}(\Omega)$. Then the restriction $f:=F_{\mid \mathbb{T}}^{*}$ is a function of bounded variation. Since $F^{*}$ is a continuous mapping in $\mathrm{cl}(\mathbb{D})$, it follows from [6, Thm. 2.11] and the maximal principle that the function $F$ can be uniquely recovered from its boundary limiting valued function $f$ by means of the Poisson integral, i.e. $F=\mathrm{P}[f]$. Therefore, we can use the relevant results from the previous sections to get these estimates.

Remark 6.2 Given a continuous injective function $f: \mathbb{T} \rightarrow \mathbb{C}$ of bounded variation assume that $F:=\mathrm{P}[f]$ is a locally injective mapping in $\mathbb{D}, \mathrm{J}[F](0)>0$ and $f(\mathbb{T})$ is the boundary of the image domain $F(\mathbb{D})$. Then $f(\mathbb{T})$ is a Jordan curve which is the boundary of the domain $\Omega:=F(\mathbb{D})$. Since the mapping $F$ is locally injective and has continuous extension $F^{*}$ to the closure $\operatorname{cl}(\mathbb{D})$ such that $F_{\mid \mathbb{T}}^{*}=f$, we conclude from the argument principle for topological mappings that $F$ is an injective mapping; cf. also [2, Thm. 2.7]. Therefore, we can use respective results from the previous sections to get the estimates (2.9), (2.15), (3.1), (3.6), (4.13) and (5.2).

Motivated by [11, Lem. 0.5, Thm. 0.6] we state the following results.

Lemma 6.3 Given $K \geq 1$ and a function $f: \mathbb{T} \rightarrow \mathbb{C}$ of bounded variation assume that $F:=\mathrm{P}[f]$ is a $K$-quasiregular mapping, $\mathrm{J}[F](0)>0$ and $F(\mathbb{D})$ is a convex domain satisfying $F(\mathbb{D}) \cap f(\mathbb{T})=\emptyset$. Then

$$
d_{f} \geq \frac{R_{1}+R_{2}}{\pi K} \tan \left(\frac{\pi}{2} \frac{R_{1}}{R_{1}+R_{2}}\right) \geq \frac{R_{1}}{2 K},
$$

for all $R_{1}, R_{2}>0$ satisfying the condition (4.14).

Proof Fix $K \geq 1, R_{1}, R_{2}>0$ and a function $f: \mathbb{T} \rightarrow \mathbb{C}$ satisfying the assumptions. If $F(0)=0$, then the inequalities (6.1) follow directly from Lemma 5.1 and Lemma 4.3; cf. the inequalities (4.9).

If $a:=F(0) \neq 0$ we can replace $f$ by $f_{a}:=f-a$. Then the function $f_{a}$ is of bounded variation. Moreover,

$$
F_{a}:=\mathrm{P}\left[f_{a}\right]=\mathrm{P}[f]-\mathrm{P}[a]=F-a \text { in } \mathbb{D},
$$

and so $F_{a}(0)=0, F_{a}$ is a $K$-quasiregular mapping, $\mathrm{J}\left[F_{a}\right](0)=\mathrm{J}[F](0)>0$ and $F_{a}(\mathbb{D})$ is a convex domain. Therefore, the mapping $f_{a}$ satisfies the inequalities in (6.1) with $f$ replaced by $f_{a}$. Since $f^{\prime}=f_{a}^{\prime}$ in $\mathbb{T}$ we obtain the inequalities (6.1) without the assumption $F(0)=0$, which completes the proof. 
Theorem 6.4 Given $K \geq 1$ and $R_{1}, R_{2}>0$ let $F$ be a $K$-quasiconformal and harmonic mapping of $\mathbb{D}$ onto a convex domain $\Omega$ such that $\mathbb{D}\left(F(0), R_{1}\right) \subset \Omega \subset$ $\mathbb{D}\left(F(0), R_{2}\right)$. Then

$$
|\partial F(\zeta)| \geq \frac{K+1}{K} \frac{R_{1}+R_{2}}{2 \pi} \tan \left(\frac{\pi}{2} \frac{R_{1}}{R_{1}+R_{2}}\right) \geq \frac{K+1}{K} \frac{R_{1}}{4}, \quad \zeta \in \mathbb{D} .
$$

Proof Fix $K, R_{1}, R_{2}$ and $F$ satisfying the assumption. Since $\Omega$ is a bounded convex domain, it is a Jordan domain bounded by a rectifiable Jordan curve $\Gamma$; cf. [4]. By assumption, $F$ is a quasiconformal mapping of $\mathbb{D}$ onto $\Omega$. Therefore, it has a uniquely determined homeomorphic extension $F^{*}$ onto the closure $\operatorname{cl}(\mathbb{D})$ and $F^{*}(\operatorname{cl}(\mathbb{D}))=$ $\operatorname{cl}(\Omega)=\Omega \cup \Gamma$; cf. [9]. From Remark 6.1 it follows that the inequalities (4.13) holds. Then applying Lemma 6.3 we see that for every $\zeta \in \mathbb{D}$,

$$
\begin{aligned}
|\partial F(\zeta)| & \geq \frac{R_{1}+R_{2}}{2 \pi} \tan \left(\frac{\pi}{2} \frac{R_{1}}{R_{1}+R_{2}}\right)+\frac{1}{2} d_{f} \\
& \geq\left(1+\frac{1}{K}\right) \frac{R_{1}+R_{2}}{2 \pi} \tan \left(\frac{\pi}{2} \frac{R_{1}}{R_{1}+R_{2}}\right) \geq\left(1+\frac{1}{K}\right) \frac{R_{1}}{4},
\end{aligned}
$$

which leads to (6.2).

Theorem 6.5 Let $F: \mathbb{D} \rightarrow \mathbb{C}$ be a locally injective harmonic mapping such that $\mathrm{J}[F](0)>0$ and $\bar{\partial} F(0)=0$. Then for every $R \in(0 ; 1)$,

$$
\begin{aligned}
|\partial F(\zeta)| & \geq \frac{\sqrt{6-2 R^{2}}}{4} \min _{z \in \mathbb{T}(0, R)}\left(\left|\frac{\partial F(z)}{\partial r}\right|^{2}+\frac{1}{R^{2}}\left|\frac{\partial F(z)}{\partial \theta}\right|^{2}\right)^{1 / 2} \\
& \geq \frac{\sqrt{\left(3-R^{2}\right)\left(1+R^{2}\right)}}{2(1+R) R} \min _{z \in \mathbb{T}(0, R)}\left|\frac{\partial F(z)}{\partial \theta}\right|, \quad \zeta \in \overline{\mathbb{D}}(0, R) .
\end{aligned}
$$

Proof Let $F$ be a mapping satisfying the assumption. As in Corollary 2.4 we see that the condition (2.16) holds, and so the second dilatation

$$
\mathbb{D} \ni \zeta \mapsto \omega(\zeta):=\frac{\overline{\bar{\partial} F(\zeta)}}{\partial F(\zeta)}
$$

of $F$ is a well-defined holomorphic function as well as

$$
|\omega(\zeta)|<1, \quad \zeta \in \mathbb{D}
$$

By assumption $\bar{\partial} F(0)=0, \omega(0)=0$. Then by Schwarz lemma $|\omega(\zeta)| \leq|\zeta|$ for $\zeta \in \mathbb{D}$. In particular, for a given $R \in(0 ; 1)$ we obtain $|\omega(\zeta)| \leq R$ for $\zeta \in \mathbb{D}(0, R)$. Therefore, the function $\mathbb{D} \ni \zeta \mapsto F_{R}(\zeta):=F(R \zeta)$ is locally injective and $K$ quasiregular in $\mathbb{D}$ with $K:=(1+R) /(1-R)$. As in the proof of Corollary 3.2 we define the function $\mathbb{T} \ni z \mapsto f(z):=F(R z)$. From (3.7) and Theorem 5.2 it follows that 


$$
\begin{aligned}
\left|\partial F_{R}(\zeta)\right| & \geq \frac{\sqrt{K^{2}+4 K+1}}{2(K+1)} \underset{z \in \mathbb{T}}{\operatorname{essinf}}\left(|\mathrm{A}[\mathrm{d} f](z)|^{2}+\left|f^{\prime}(z)\right|^{2}\right)^{1 / 2} \\
& \geq \frac{\sqrt{\left(K^{2}+4 K+1\right)\left(K^{2}+1\right)}}{2(K+1) K} d_{f} .
\end{aligned}
$$

Combining this with (3.8), (3.9) and (3.10) we obtain the estimates (6.3), which proves the theorem.

Remark 6.6 An easy computation shows that for every differentiable function $F$ : $\mathbb{D} \rightarrow \mathbb{C}$,

$$
\left|\partial_{x} F(\zeta)\right|^{2}+\left|\partial_{y} F(\zeta)\right|^{2}=2\left(|\partial F(\zeta)|^{2}+|\bar{\partial} F(\zeta)|^{2}\right) \geq 2|\partial F(\zeta)|^{2}, \quad \zeta \in \mathbb{D} .
$$

Therefore, any lower estimate of $|\partial F|$ in $\mathbb{D}$ leads to one of $\left|\partial_{x} F\right|^{2}+\left|\partial_{y} F\right|^{2}$ in $\mathbb{D}$. For example, under the assumptions of Theorem 4.4 the estimate

$$
\begin{aligned}
\left|\partial_{x} F(\zeta)\right|^{2}+\left|\partial_{y} F(\zeta)\right|^{2} & \geq 2\left(\frac{R_{1}+R_{2}}{2 \pi} \tan \left(\frac{\pi}{2} \frac{R_{1}}{R_{1}+R_{2}}\right)+\frac{1}{2} d_{f}\right)^{2} \\
& \geq 2\left(\frac{R_{1}}{4}+\frac{1}{2} d_{f}\right)^{2}, \quad \zeta \in \mathbb{D}
\end{aligned}
$$

holds for all $R_{1}, R_{2}>0$ satisfying the condition (4.14). If additionally $F$ is a $K$ quasiconformal mapping for a given $K \geq 1$, we conclude from (6.4) and Theorem 6.4 that

$$
\begin{aligned}
\left|\partial_{x} F(\zeta)\right|^{2}+\left|\partial_{y} F(\zeta)\right|^{2} & \geq 2\left(\frac{K+1}{K} \frac{R_{1}+R_{2}}{2 \pi} \tan \left(\frac{\pi}{2} \frac{R_{1}}{R_{1}+R_{2}}\right)\right)^{2} \\
& \geq 2\left(\frac{K+1}{K} \frac{R_{1}}{4}\right)^{2}, \quad \zeta \in \mathbb{D} .
\end{aligned}
$$

Open Access This article is distributed under the terms of the Creative Commons Attribution License which permits any use, distribution, and reproduction in any medium, provided the original author(s) and the source are credited.

\section{References}

1. Astala, K., Iwaniec, T., Martin, G.: Elliptic Partial Differential Equations and Quasiconformal Mappings in the Plane. Princeton Mathematical Series, Princeton University Press, Princeton and Oxford (2009)

2. Bshouty, D., Hengartner, W.: Univalent harmonic mappings in the plane. Ann. Univ. Mariae CurieSkłodowska Sect. A 48, 12-42 (1994)

3. Duren, P.: Theory of $H^{p}$-Spaces. Dover Publications Inc., Mineola, New York (2000)

4. FitzGerald, C.H., Lesley, F.D.: Integrability of the derivative of the Riemann mapping function for wedge domains. J. D’Analyse Math. 49, 271-292 (1987)

5. Garnett, J.B.: Bounded Analytic Functions. Academic Press, New York (1981)

6. Hayman, W.K., Kennedy, P.B.: Subharmonic Functions, vol. I. Academic Press, London (1976) 
7. Heinz, E.: On one-to-one harmonic mappings. Pac. J. Math. 9, 101-105 (1959)

8. Kalaj, D.: On harmonic diffeomorphisms of the unit disc onto a convex domain. Complex Var. 48(2), 175-187 (2003)

9. Lehto, O., Virtanen, K.I.: Quasiconformal Mappings in the Plane, 2nd ed. Grundlehren 126. Springer, Berlin (1973)

10. Lewy, H.: On the non-vanishing of the Jacobian in certain one-to-one mappings. Bull. Am. Math. Soc. 42, 689-692 (1936)

11. Partyka, D., Sakan, K.: On Heinz’s inequality. Bull. Soc. Sci. Lettres Łódź 52, 27-34 (2002), Série: Recherches sur les déformations 36

12. Partyka, D., Sakan, K.: On an asymptotically sharp variant of Heinz's inequality. Ann. Acad. Sci. Fenn. Ser. A. I. Math. 30, 167-182 (2005)

13. Partyka, D., Sakan, K.: On a variant of Heinz's inequality for harmonic mappings of the unit disk onto bounded convex domains. Bull. Soc. Sci. Lett. Łódź 59, 25-36 (2009), Série: Recherches sur les déformations 59(2)

14. Partyka, D., Sakan, K.: Quasiconformal and Lipschitz Harmonic Mappings of the Unit Disk Onto Bounded Convex Domains. In submission

15. Pommerenke, C.: Boundary Behaviour of Conformal Maps. Springer, Berlin (1992) 